\title{
Improving Light Extraction From Heavy Inorganic Scintillators by Photonic Crystals
}

\author{
Matthias Kronberger, Etiennette Auffray, Member, IEEE, and Paul R. Lecoq, Member, IEEE
}

\begin{abstract}
The impact of photonic crystal (PhC) slabs on the extraction of light from the heavy inorganic scintillators LuYAP and LYSO is evaluated by combining numerical transmission calculations of a scintillator with $\mathrm{PhC}$ coupling face with simulations of the light propagation inside the scintillator. The transmission of the scintillator-PhC coupling face is determined by means of a scattering-matrix algorithm. The $\mathrm{PhC}$ slab is assumed to consist of a bulk material with a triangular pattern of air holes that is sandwiched between the scintillator substrate and a layer of optical grease. By folding these data with the angular distribution of the scintillation photons arriving at the coupling face, the light collection efficiency $\eta_{L}$ of the system is estimated. The results indicate that a scintillator coupling face equipped with a PhC slab can exhibit a significant gain in $\eta_{L}$. This gain is due to the extraction of photons that are lost in a scintillator with plain exit surface due to total internal reflection. The largest simulated gains of up to a factor of two are observed for small scintillators and for PhC coupling faces with $n_{\text {bulk }} \ll n_{\text {sub }}, n_{\text {avg }} \approx \sqrt{n_{\text {sub }} \cdot n_{\text {grease }}}$, and $d \leq a$, where $n_{\text {bulk }}, n_{\text {sub }}$ and $n_{\text {grease }}$ are the respective refractive indexes of the $\mathrm{PhC}$ bulk, the scintillator substrate, and the optical grease, $a$ the lattice constant of the PhC pattern, $d$ the thickness of the PhC slab, and $n_{\text {avg }}$ the average refractive index of the PhC slab determined by $n_{\text {bulk }}$ and the filling factor $f$. Due to the approximations and idealizations of the model, these gains in light collection efficiency may be lower in practical applications and are expected to be achieved only with specular reflectors with reflectivities above $90 \%$, and $\mathrm{PhC}$ bulk materials with absorption coefficients $\alpha_{\text {abs }} \leq 10^{3}-10^{4} \mathrm{~cm}^{-1}$ over the whole wavelength range of emission of the scintillator.
\end{abstract}

Index Terms-Inorganic scintillators, light extraction, LSO, LuAP, photonic crystals, scintillation yield.

\section{INTRODUCTION}

$\mathbf{I}$ NORGANIC scintillators are widely used in modern medical imaging modalities as a converter for the kiloelectronvolt $(\mathrm{keV})$ and microelectronvolt $(\mathrm{MeV})$ photons that are employed in these systems to obtain information about morphology and metabolism of the examined body. Likewise, they are applied in high-energy physics to measure the energy of particles that are ejected from the interaction point of a collider or a fixed-target experiment. In both cases, their use is motivated by

Manuscript received May 12, 2009; revised September 10, 2009, February 25, 2010, and July 06, 2010; accepted July 14, 2010. Date of current version October 15, 2010. This work was performed in the framework of the Crystal Clear Collaboration.

The authors are with the European Centre for Nuclear Research CERN, 1211 Geneva 23, Switzerland (e-mail: matthias.kronberger@cern.ch; etiennette.auffray@cern.ch; paul.lecoq@cern.ch).

Color versions of one or more of the figures in this paper are available online at http://ieeexplore.ieee.org.

Digital Object Identifier 10.1109/TNS.2010.2063438 the very good detection efficiency of modern scintillation detectors for hard radiation. This allows the construction of relatively compact and highly pixelized systems with a good spatial resolution.

One of the key problems in the development of next-generation particle physics experiments and medical imaging systems is the optimization of the energy resolution $\Delta E$ and the timing resolution $\Delta t$ of the detectors. Both parameters are strongly influenced by the statistical fluctuations of the number of photoelectrons $N_{p e}$ registered after a particle has deposited its energy in the scintillator. Typically, $N_{p e}$ is expressed as

$$
N_{p e}[\mathrm{pe} / \mathrm{MeV}]=q_{\mathrm{eff}} \cdot L Y=q_{\mathrm{eff}} \cdot \eta_{L} \cdot L Y_{\mathrm{abs}}
$$

where $q_{\text {eff }}$ is the effective quantum efficiency of the photodetector, $L Y$ the number of scintillation photons arriving at the photocathode per $\mathrm{MeV}$ of absorbed radiation, and $L Y_{\text {abs }}$ the number of photons produced in the scintillator per $\mathrm{MeV}$ of absorbed radiation. More commonly, $L Y$ and $L Y$ abs are referred to as light output and absolute light yield of the scintillator, respectively. The parameter $\eta_{L}=L Y / L Y_{\mathrm{abs}}$ is the light collection efficiency of the scintillation detector and summarizes the impact of all processes which lead to losses during the transport of the scintillation photons from the production point to the active region of the photodetector. This includes absorption and scattering in the scintillator substrate, absorption and diffusion at enveloping materials, and reflection losses due to mismatch of the refractive indexes of scintillator, optical coupling, and photodetector. Especially in tiny detector crystals with small aspect ratio, $\eta_{L}$ is small and a large fraction of the emitted photons is lost during the transport to the photodetector. These losses increase the statistical fluctuations of the light output and therefore degrade $\Delta E$ and $\Delta t$.

In recent years, micro- and nanostructurization of materials have found widespread interest as a tool to overcome the problem of total internal reflection, which is a major source of losses in light-emitting and -transmitting media with high refractive index. A rather novel technique is the use of thin photonic crystal $(\mathrm{PhC})$ slabs-media with a periodic modulation of the dielectric constant $\epsilon$ [1], [2]—to guide incident photons into or out of the substrate. In an earlier publication [3], the principal physical processes that govern the extraction of light from a scintillator that is equipped with a $\mathrm{PhC}$ slab have been qualitatively reviewed. The present work evaluates the impact of various structural parameters of a $\mathrm{PhC}$ on light extraction and presents a simple approach which allows for estimating the effect of the $\mathrm{PhC}$ on $\eta_{L}$ for a given scintillator. The main focus of the study is on the two heavy inorganic scintillators LuYAP and LYSO that have been used in the dedicated PET systems 
ClearPET and ClearPEM developed by the Crystal Clear Collaboration [4], [5]; however, the results presented herein are also valid for many other scintillators with comparable optical properties.

\section{MATERIALS AND MethodS}

The light collection efficiency of a scintillation detector that contains a scintillator with $\mathrm{PhC}$ coupling face is mainly governed by three parameters: 1) the angular distribution of the scintillation photons incident on the coupling face, 2) the dependence of the $\mathrm{PhC}$ transmission on the incidence angle of the photons, and 3) the probability that a photon that is reflected or deflected back into the scintillator arrives again at the coupling face. Moreover, it has to be taken into account that scintillation photons are not emitted at a single wavelength but over a range of wavelengths due to the broadening of the electronic states by the crystal field; this implies that an optimization of $\eta_{L}$ can only be achieved if the transmission of the PhC slab is sufficiently high over the whole emission range of the scintillator.

\section{A. Simulation of PhC Transmission}

The transmission of the $\mathrm{PhC}$ exit surface was calculated by applying the scattering matrix method of Tikhodeev et al. [6] and adapting it to the current problem. The simulated model structure (Figs. 1 and 2) consists of an LYSO or LuYAP type substrate, a PhC slab consisting of a regular pattern of air holes, and a layer of grease. All simulated materials were assumed to be ideally transparent. The refractive index of the grease, $n_{\text {grease }}$, was set to 1.47 . For the substrate, refractive indexes $n_{\text {sub }}$ of 1.82 and 1.94 were used to simulate LYSO and LuYAP, respectively. The $\mathrm{PhC}$ slab was simulated as a slab of thickness $d$ containing a triangular arrangement of air holes $\left(n_{\text {holes }}=1.0\right)$ with lattice constant $a$ and filling factor $f$, where $f$ expresses the fraction of the PhC surface occupied by the air holes. To eliminate any wavelength dependence of the results, $a$ was normalized to the wavelength $\lambda$ of the incident photon and only the ratio $a / \lambda$ was varied. For the refractive index of the $\mathrm{PhC}$ bulk material, $n_{\text {bulk }}$, a wide range of values between $n_{\text {bulk }}=n_{\text {sub }}$ and $n_{\text {bulk }}=3.6$ was used, which corresponds to the refractive index range within which the majority of candidate bulk materials are situated (see Section III-A for a discussion of possible PhC slab materials). Further tests were performed for a few selected values up to $n_{\text {bulk }}=4.8$, which is approximately the refractive index of elemental silicon at the maximum emission wavelength of LYSO of $420 \mathrm{~nm}$ [7], [8]. The parameters $f$ and $d$ were varied over a wide range $(0.2 a \leq d \leq 2.0 a$; $0.2 \leq f \leq 0.8$ ) to identify $\mathrm{PhC}$ configurations with promising transmission properties for a given value of $n_{\text {bulk }}$. In each simulation run, between 85 and 151 plane waves were simulated; these numbers were found to be sufficient for assessing the principal optical properties of the material. For the determination of the $\mathrm{PhC}$ transmission, it had to be taken into account that a) the scintillation photons are generally emitted unpolarized relative to the $\mathrm{PhC}$ slab, and b) that the transmission of the $\mathrm{PhC}$ slab depends on the orientation of the electric field vector relative to the periodicity plane. For this reason, the incident photons were assumed to be superpositions of plane waves with the electric field vector in the $x-y$ plane (S polarized waves) and

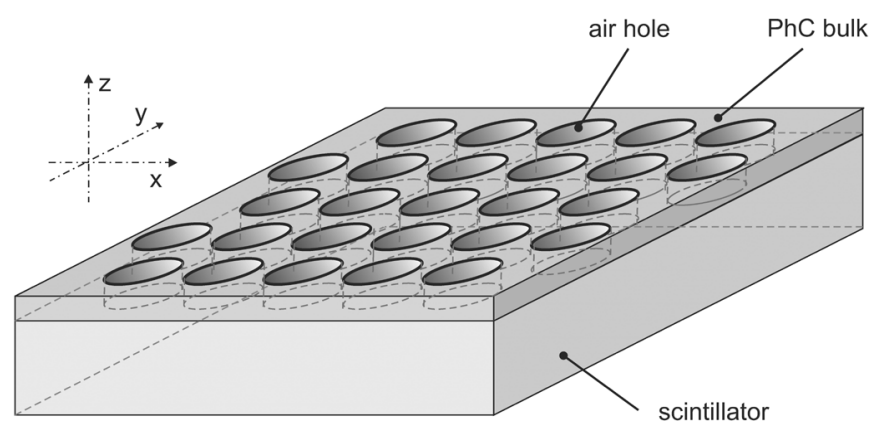

Fig. 1. Outline of the simulated system in oblique projection. The scintillator substrate, $\mathrm{PhC}$ bulk, and the triangular air hole pattern are indicated in the drawing. For reasons of clarity, the layer of grease on top of the $\mathrm{PhC}$ is not shown.

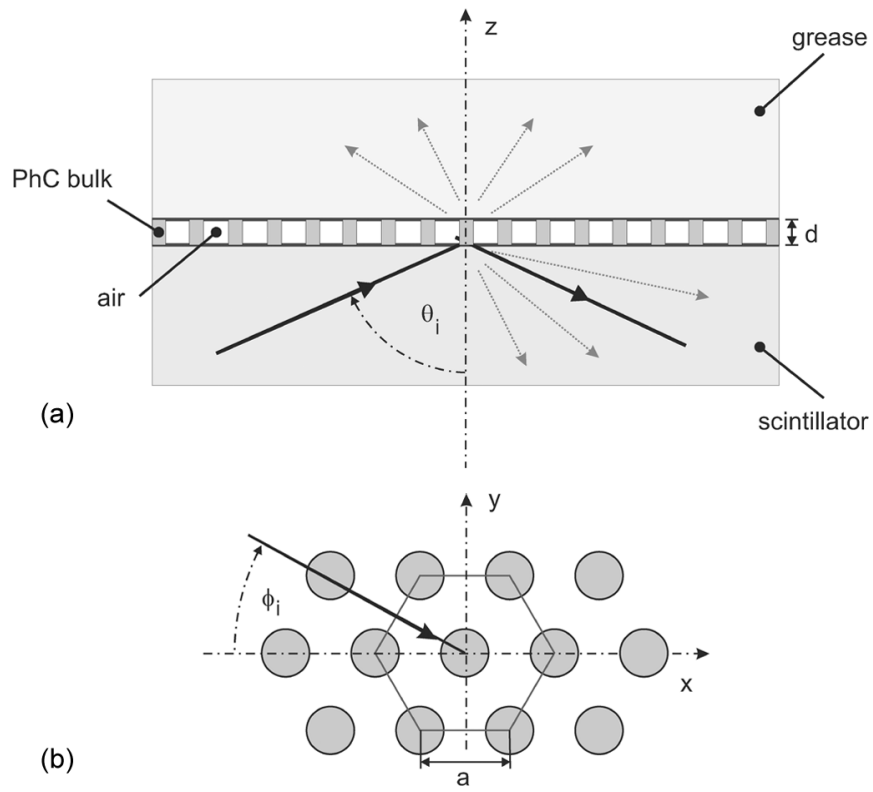

Fig. 2. (a) Front and (b) top view of the simulated system. The definitions of the angles $\theta_{i}$ and $\phi_{i}$, the slab thickness $d$, and the lattice constant $a$ are indicated in the plots.

waves with the magnetic field vector in the $x-y$ plane (P polarized waves). Accordingly, the transmission of the $\mathrm{PhC}$ slab per incidence angle $\theta_{i}$ and polar angle $\phi_{i}$ was determined by averaging over the transmissions calculated for both polarization states. Finally, the total transmission of the PhC exit surface was determined by integrating over $\theta_{i}$ and $\phi_{i}$.

\section{B. Determination of the Angular Distribution of Incoming Photons}

The angular distribution of the photons arriving at the exit surface of the scintillator was determined with the light ray tracing program LITRANI [9]. The simulated setup comprised a LuYAP or LYSO scintillator enveloped in a reflecting material, a photomultiplier (PMT) with fused silica entry window, and a slice of grease between scintillator and PMT. The scintillation light was generated by $662 \mathrm{keV} \gamma$-photons originating from a ${ }^{137} \mathrm{Cs} \gamma$-source that was positioned in $0.5 \mathrm{~cm}$ distance from the scintillator opposite to the coupling face. The photoabsorption and Compton scattering coefficients of LYSO and LuYAP were taken from the NIST database [10]. To achieve a realistic modelling of the photon propagation in the scintillator, the refractive 


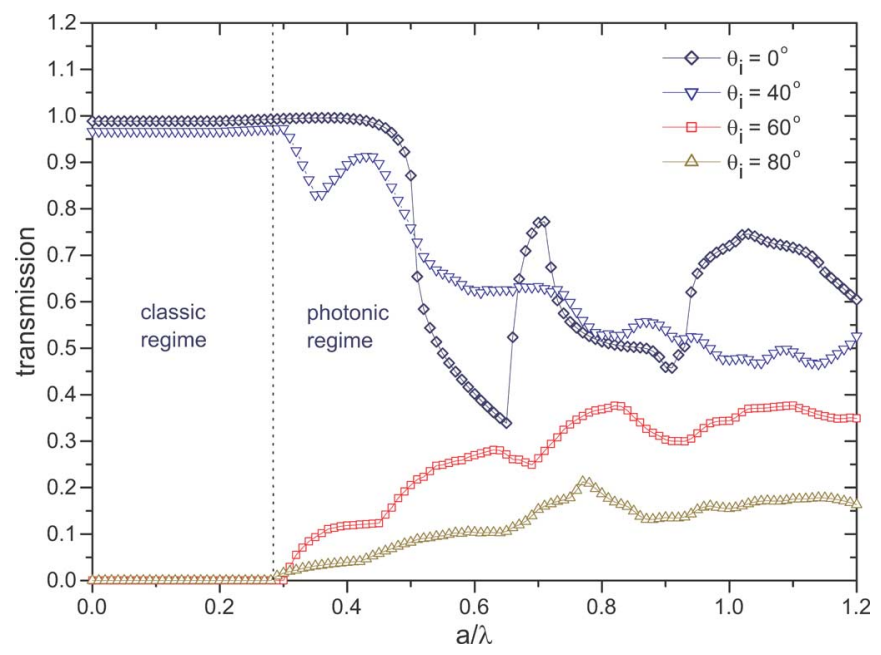

Fig. 3. Dependence of the slab transmission on the incidence angle $\theta_{i}$ of the incoming photons for a LuYAP scintillator with PhC coupling face and $n_{\text {bulk }}=$ $3, d=0.3 a$, and $f=0.7$.

indices, attenuation coefficients $\alpha_{\text {att }}$, and emission characteristics were taken from laboratory measurements of real LYSO and LuYAP samples [11]. Photon scattering within the scintillator volume was taken into account by introducing a parameter $B$ which is given by [12]

$$
B=\frac{\alpha_{\text {scat }}}{\alpha_{\text {abs }}}
$$

where $\alpha_{\text {abs }}$ and $\alpha_{\text {scat }}$ are the absorption and scattering coefficients, respectively, and $\alpha_{\text {att }}=\alpha_{\text {scat }}+\alpha_{\text {abs }}$. Following the results of earlier studies [13], $B$ was assumed to be 0.1 and 1.6 for LuYAP and LYSO, respectively. In contrast to the realistic modelling of the light propagation inside the scintillators, the reflector enveloping the scintillator was assumed to be purely specular with an ideal reflectivity of $100 \%$. Although this assumption contradicts the intention of a realistic simulation to some extent, it made it possible to evaluate how many photons could be extracted from a realistic scintillator in the best of cases.

\section{DISCUSSION OF RESULTS}

The dependence of the $\mathrm{PhC}$ slab transmission on the incidence angle $\theta_{i}$ is depicted in Fig. 3 for a LuYAP scintillator equipped with a PhC slab with $n_{\text {bulk }}=3 d=0.3 a$ and $f=0.7$. Using the definition of $\theta_{i}$ from Fig. $2, \theta_{i}=0^{\circ}$ corresponds to normal incidence on the $\mathrm{PhC}$ slab, while $\theta_{i}=90^{\circ}$ corresponds to a wave that travels parallel to the interface $\mathrm{PhC}$ - scintillator.

The shape of the curves clearly indicates that the transmission of the simulated structure depends not only on $\theta_{i}$ but also on $a / \lambda$. Two general regimes with fundamentally different behavior of the PhC coupling face can be distinguished: for $a / \lambda \ll$ 1 (the classic regime), the incident photon cannot resolve the periodic modulation of the $\mathrm{PhC}$ and sees the $\mathrm{PhC}$ only as a uniform slab with an average refractive index $n_{\text {avg }}$,

$$
n_{\mathrm{avg}}=(1-f) \cdot n_{\mathrm{bulk}}+f \cdot n_{\mathrm{hole}} .
$$

Since $d$ is in our case of the same order as $a$ and $a / \lambda \ll 1$, also $d \ll \lambda$ and the propagation of the photon can be described in good approximation by classical optics. Accordingly, total reflection is observed for incidence angles $\theta_{i}$ that are larger than the angle of total reflection $\theta_{\text {tot }}$ of the scintillator-grease interface,

$$
\theta_{\text {tot }}=\arcsin \left(\frac{n_{\text {grease }}}{n_{\text {sub }}}\right) .
$$

For a plain coupling face and $n_{\text {grease }}=1.47, \theta_{\text {tot }}$ is of the order of $50^{\circ}$ and $55^{\circ}$ for LuYAP and LYSO, respectively.

The simulated structure exhibits a different behavior when $\lambda$ and $a$ are of the same order. In this regime (hereafter called photonic regime), the periodic modulation of the $\mathrm{PhC}$ slab splits the incoming electromagnetic wave into a number of harmonics for which the $\mathrm{z}$ component of the wave vector $\vec{k}$ becomes

$$
k_{z, \text { grease }}=\sqrt{n_{\text {grease }}^{2} \cdot k^{2}-\left(\vec{k}_{\|}+\vec{G}\right)^{2}}
$$

in the layer of grease and

$$
k_{z, \mathrm{sub}}=\sqrt{n_{\mathrm{sub}}^{2} \cdot k^{2}-\left(\vec{k}_{\|}+\vec{G}\right)^{2}}
$$

in the substrate, where $\vec{G}$ is a reciprocal vector of the PhC lattice and $\vec{k}_{\|}$the in-plane component of the wave vector $k=2 \pi / \lambda$ of the incoming photon given by

$$
\vec{k}_{\|}=n_{\mathrm{sub}} \cdot k \cdot \sin \theta_{i} \cdot\left(\cos \phi_{i}, \sin \phi_{i}\right) \text {. }
$$

Depending on whether $k_{z \text {,grease }}$ is real for a harmonic or not, it is transmitted into the layer of grease or reflected back into the substrate. Accordingly, the transmission properties of the slab change in the photonic regime, and pronounced maxima and minima are observed where new harmonics are transmitted into the layer of grease or reflected back into the scintillator substrate. Generally, this leads to a reduction of the slab transmission for $\theta_{i}<\theta_{\text {tot }}$ since more power is reflected back into the substrate than in the case of a plain exit surface; for $\theta_{i}>\theta_{\mathrm{tot}}$, on the other hand, non-zero transmission is observed because $k_{z \text {,grease }}$ reaches a real value for some of the harmonics. Especially this latter point is significant in our context since a large fraction of the scintillation photons arrives at the coupling face under an incidence angle that is larger than $\theta_{\text {tot }}$ [3]. The minimum value of $|\vec{k}|$ for which an additional harmonic is coupled into the layer of grease depends on $\theta_{i}$ and is given by

$$
|\vec{k}|_{\min }=(a / \lambda)_{\min }=\left(n_{\mathrm{sub}} \cdot \sin \theta_{i}+n_{\text {grease }}\right)^{-1}
$$

For grazing incidence $\left(\theta_{i} \approx 90^{\circ}\right),(a / \lambda)_{\min }$ becomes 0.30 for LYSO and 0.29 for LuYAP, which are also the respective lower limits of the photonic regime if scattering into the substrate is not considered.

The dependence of the slab transmission on the bulk refractive index $n_{\text {bulk }}$ and the structural parameters of the PhC is depicted in Figs. 4 and 5. Each plot shows the transmission $t$ for 


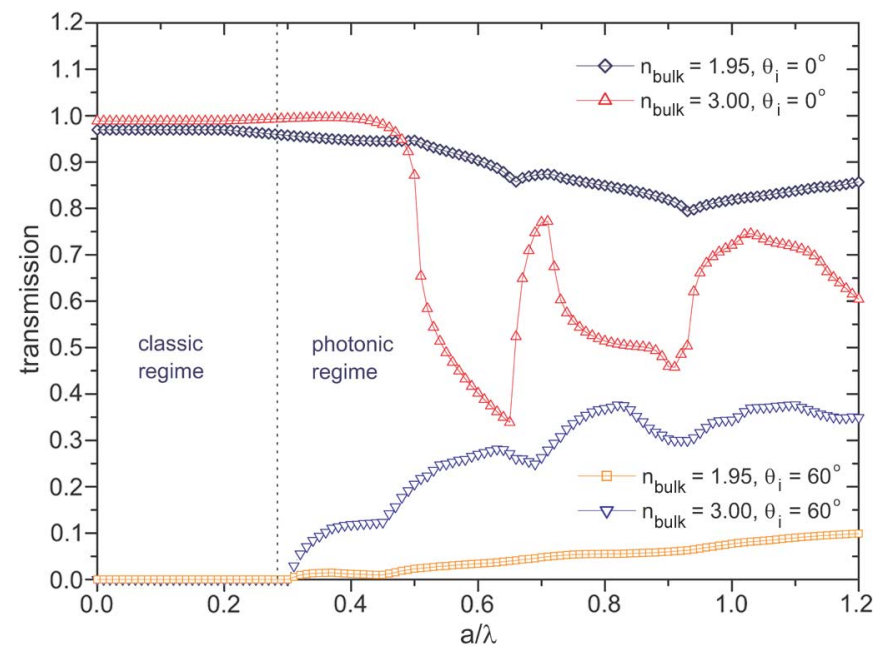

Fig. 4. Dependence of the slab transmission on $n_{\text {bulk }}$ for a LuYAP scintillator with PhC coupling face. Transmission curves are shown for two different values of $n_{\text {bulk }}$ and for incidence angles $\theta_{i}=0^{\circ}$ and $\theta_{i}=60^{\circ}$. In both cases, $d=$ $0.3 a$ and $f=0.7$. Note that the angle of total reflection of the given structure, $\theta_{\text {tot }}$, is $50^{\circ}$ for $a / \lambda \ll 1$.

two values of the respective parameter and for incidence angles $\theta_{i}=0^{\circ}$ and $\theta_{i}=60^{\circ}$. Clearly, all three parameters affect the transmission properties of the coupling face in a significant manner. In particular, the plots show that a change of the value of a parameter that leads to an increase in $t$ for $\theta_{i}<\theta_{\text {tot }}$ often leads to a decrease of $t$ for $\theta_{i}>\theta_{\text {tot }}$, and vice versa. Due to the aforementioned large fraction of scintillation photons with $\theta_{i}>\theta_{\text {tot }}$, clearly those parameter combinations have the potential to optimize $\eta_{L}$ where $t$ is sufficiently large for this angular range. Still, a tuning of the $\mathrm{PhC}$ coupling face for a given scintillator can only be achieved when the angular distribution of the incident scintillation photons is taken into account as a whole.

Fig. 6 compares, for $2 \times 2 \times 8 \mathrm{~mm}^{3} \mathrm{LuYAP}$ and LYSO scintillators, the angular distribution of the photons that are extracted at the $\mathrm{PhC}$ coupling face immediately (that is, at first incidence of the photon onto the $\mathrm{PhC}$ ) with the angular distribution of the photons extracted from a scintillator with plain exit surface. In both cases, $n_{\text {bulk }}$ was set to 3 . In the case of LuYAP, $d$ and $f$ were set to $d=0.5 a$ and $f=0.7$ whereas $d=0.3 a$ and $f=0.7$ was used in the case of LYSO. In both cases, $a / \lambda$ was set to 0.8 , which is well inside the photonic regime of the $\mathrm{PhC}$ coupling face. As the plot shows, the nanopatterned exit surface has a reduced transmission at angles below the angle of total reflection $\theta_{\text {tot }}$, and the total number of photons transmitted in this angular region is clearly smaller than that observed for a plain exit surface. However, the plots also show that the losses at $\theta_{i}<\theta_{\text {tot }}$ are at least to a part compensated by the extraction of photons with $\theta_{i}>\theta_{\text {tot }}$.

These considerations concern only those photons which are transmitted at first incidence at the PhC coupling face. However, as noted before a full assessment of the light extraction potential of the scintillator-PhC system is only possible when the photons that are reflected back into the scintillator are also taken into account. These photons can be extracted at a following incidence onto the $\mathrm{PhC}$ coupling face and can add significantly to the total light output of the scintillator.
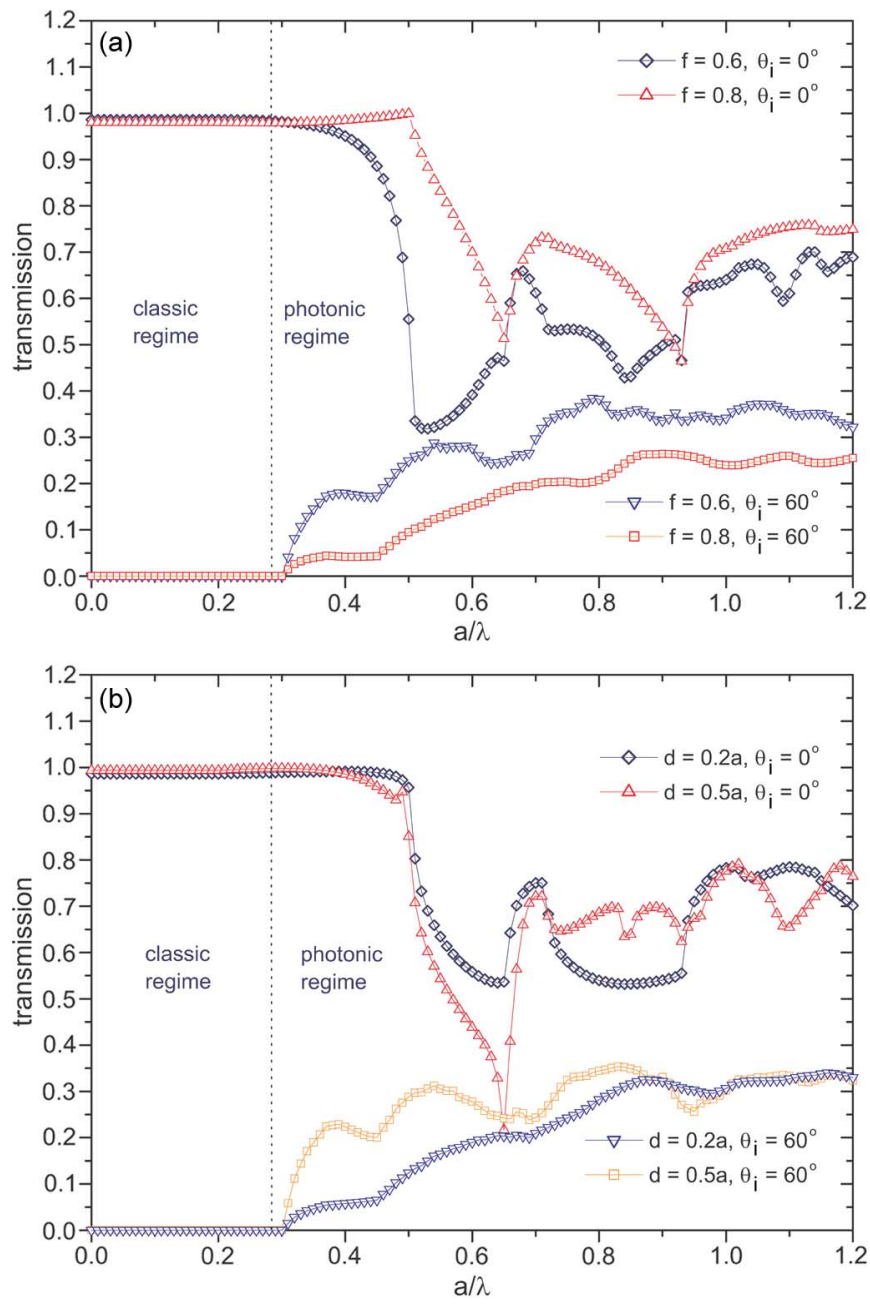

Fig. 5. Dependence of the slab transmission on (a) filling factor $f$ and (b) slab thickness $d$ for a LuYAP scintillator with PhC coupling face. Transmission curves are shown for two different values of each parameter and for incidence angles $\theta_{i}=0^{\circ}$ and $\theta_{i}=60^{\circ}$. In case (a), $d=0.3$; in case (b), $f=0.7$. $n_{\text {bulk }}$ was set to a value of 3 in both cases. Note that the angle of total reflection of the given structure, $\theta_{\text {tot }}$, is $50^{\circ}$ for $a / \lambda \ll 1$.

A relatively simple method to evaluate the impact of a $\mathrm{PhC}$ on $\eta_{L}$ is to calculate the number of extracted photons by a model that is based on the following assumptions:

1) $\alpha_{\text {abs }}$ of the scintillator is independent on the wavelength $\lambda$;

2) no scattering in the scintillator volume $\left(\alpha_{\text {scat }}=0\right)$;

3 ) the enveloping material has perfect reflectivity;

4) a lossless $\mathrm{PhC}$ bulk material $(k=0)$;

$5)$ the number of photons reflected into one direction, $N_{r}\left(\theta_{i}\right)$ is equal to the number of incident photons in this direction $N_{i}\left(\theta_{i}\right)$ reduced by the number of transmitted photons: $N_{r}\left(\theta_{i}\right)=\left(1-t\left(\theta_{i}\right)\right) N_{i}\left(\theta_{i}\right)$.

$t\left(\theta_{i}\right)$ denotes the transmission of the $\mathrm{PhC}$ slab at a given incident angle and depends on $a / \lambda$.

The assumptions quoted above have several consequences. Assumption 1) means that the absorption characteristics of the scintillator are described by a single absorption coefficient $\alpha_{\text {abs }}$. This condition is necessary as the model seeks to find an optimized value of $a / \lambda$ for the PhC structure and any other wavelength dependence is not considered. Assumptions 2) and 3) 


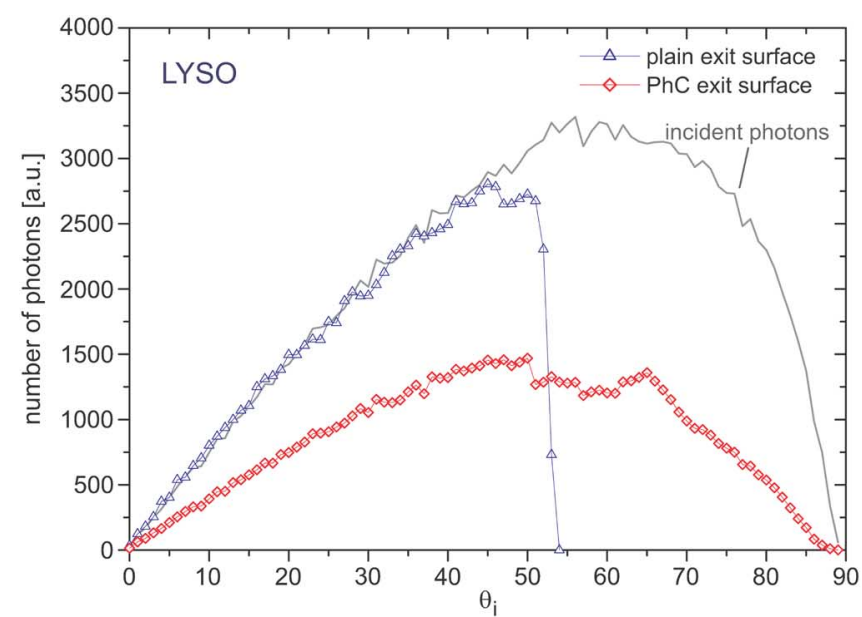

(a)

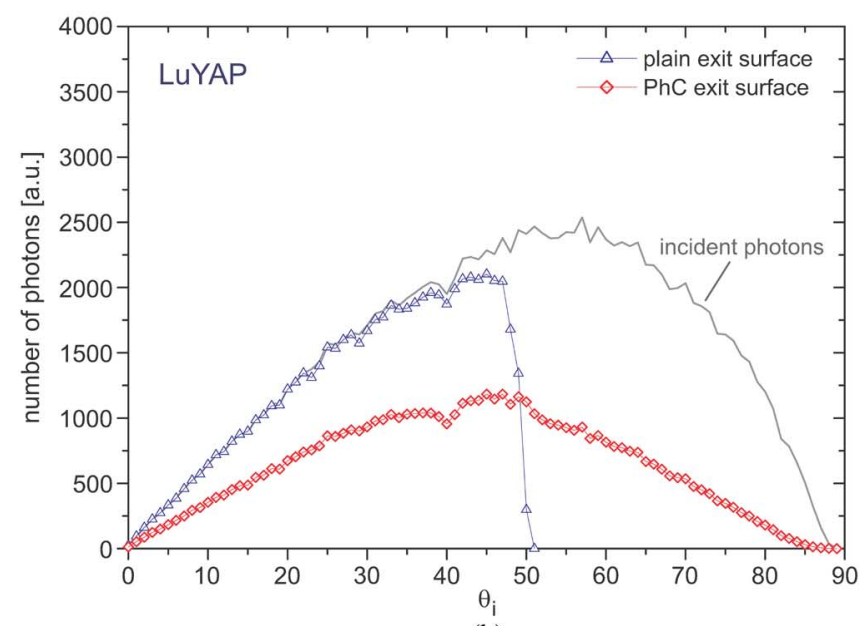

(b)

Fig. 6. Angular distribution of photons extracted at first incidence from a $2 \times$ $2 \times 8 \mathrm{~mm}^{3}$ scintillator with $\mathrm{PhC}$ equipped coupling face. The gray line indicates the angular distribution of all incident photons for a specular reflector with $100 \%$ reflectivity. (a) LuYAP, $n_{\text {bulk }}=3, d=0.5 a, f=0.7$; (b) LYSO, $n_{\text {bulk }}=3$, $d=0.3 a, f=0.7$. In both cases, the normalized lattice constant $a / \lambda$ was set to 0.8 . The angular distribution of photons extracted from a $2 \times 2 \times 8 \mathrm{~mm}^{3}$ scintillator with plain exit surface is shown for comparison.

imply that the path length $l$ travelled by a photon between reflection and recurrence at the $\mathrm{PhC}$ coupling face depends only on the incident angle $\theta_{i}$ and the size $h$ of the scintillator perpendicular to the coupling face. Therefore, $l$ can be expressed by

$$
l=2 h / \cos \theta_{i}
$$

and the probability of recurrence of the photon at the coupling face, $P_{\text {re }}\left(\theta_{i}\right)$, is only determined by $l$ and the absorption coefficient $\alpha_{\text {abs }}$ :

$$
P_{\mathrm{re}}\left(\theta_{i}\right)=e^{-l \cdot \alpha_{\mathrm{abs}}}=e^{-\frac{2 h \cdot \alpha_{\mathrm{abs}}}{\cos \theta_{i}}} .
$$

Assumption 4) specifies that absorption in the $\mathrm{PhC}$ bulk can be neglected. Finally, Assumption 5) means that the splitting of the reflected wave into several modes does not have any influence on the angular distribution of the reflected photons. This does not necessarily mean that the splitting itself is neglected; instead, it means that the angular distribution of the reflected harmonics is identical to the angular distribution of the reflected waves immediately before hitting the exit surface.

With these assumptions, the total transmission per unit of angle $T\left(\theta_{i}\right)$ is determined by

$$
T\left(\theta_{i}\right)=t\left(\theta_{i}\right) \cdot \frac{1}{1-\left(1-t\left(\theta_{i}\right)\right) \cdot e^{-\frac{2 h \cdot \alpha_{a b s}}{\cos \theta_{i}}} .}
$$

As $t\left(\theta_{i}\right)$ depends on $a / \lambda, T\left(\theta_{i}\right)$ is also a function of $a / \lambda$. The light collection efficiency $\eta_{L}$ can then be calculated by the integral

$$
\eta_{L}=\int_{0}^{\frac{\pi}{2}} T\left(\theta_{i}\right) \cdot P_{i}\left(\theta_{i}\right) \cdot d \theta_{i}
$$

where $P_{i}\left(\theta_{i}\right)$ is the probability that a photon produced in a scintillation event at an angle $\theta_{i}$ arrives at least once at the coupling face.

Fig. 7(a) and (b) depicts the angular distribution of the extracted photons for the same two systems as in Fig. 6. In the case of LuYAP, $\alpha_{\text {abs }}$ was set to $0.1 \mathrm{~cm}^{-1}$ while for LYSO, $\alpha_{\mathrm{abs}}=0.05 \mathrm{~cm}^{-1}$. Clearly, the number of extracted photons, as determined by the model presented above, is significantly higher for the scintillator with $\mathrm{PhC}$ coupling face than for the scintillator with plain exit surface. In fact, the $\mathrm{PhC}$ coupling face works so well that the majority of the photons that arrive at the coupling face are extracted.

Fig. 8(a) and (b) illustrates the dependence of $\eta_{L}$ on $a / \lambda$ for $2 \times 2 \times 8 \mathrm{~mm}^{3}$ LuYAP and LYSO scintillators and several scintillator-PhC combinations. Both figures show clearly the large increase of $\eta_{L}$ between the classic and the photonic regime. In the photonic regime, $\eta_{L}$ remains at a constant high level and only small fluctuations with $a / \lambda$ are observed. This is mainly a consequence of the high recurrence probability of the photons which makes the number of extracted photons per unit of angle only weakly dependent on $t\left(\theta_{i}\right)$. The highest gains are observed for PhC coupling faces with $n_{\text {bulk }} \gg n_{\mathrm{sub}}, n_{\mathrm{avg}} \approx$ $\sqrt{n_{\text {sub }} \cdot n_{\text {grease }}}$, and $d \leq a$ and amount to $\approx 50 \%-60 \%$ for LuYAP and $80 \%-100 \%$ for LYSO. After all, the insensitivity of $\eta_{L}$ on $a / \lambda$ is important in our context as scintillators emit over a wide wavelength range of up to several hundred nanometers and any strong fluctuations of $\eta_{L}$ with $a / \lambda$ would make the choice of a suitable lattice constant very difficult.

The dependence of $\eta_{L}$ on the scintillator dimensions is depicted in Fig. 9 for LuYAP with a PhC coupling face with $n_{\text {bulk }}=3, f=0.7$ and $d=0.5 a$. In all cases, the PhC slab is contained in one of the $2 \times 2 \mathrm{~mm}^{2}$ surfaces. The figure shows that the gain in $\eta_{L}$ decreases from almost $75 \%$ for the smallest scintillator to $\approx 50 \%$ for the largest one. This decrease is due to a changing angular distribution of the incoming photons: While the path length is only moderately increasing for photons travelling at small $\theta_{i}$, it increases dramatically for photons with large $\theta_{i}$. As a consequence, the number of photons arriving at the coupling face decreases much stronger for large $\theta_{i}$ than for small $\theta_{i}$, which effectively reduces the pool of photons with $\theta_{i}>\theta_{\text {tot }}$.

On the other hand, the reflectivity $\rho$ of the wrapping has only modest influence on the number of extracted photons (Fig. 10). 


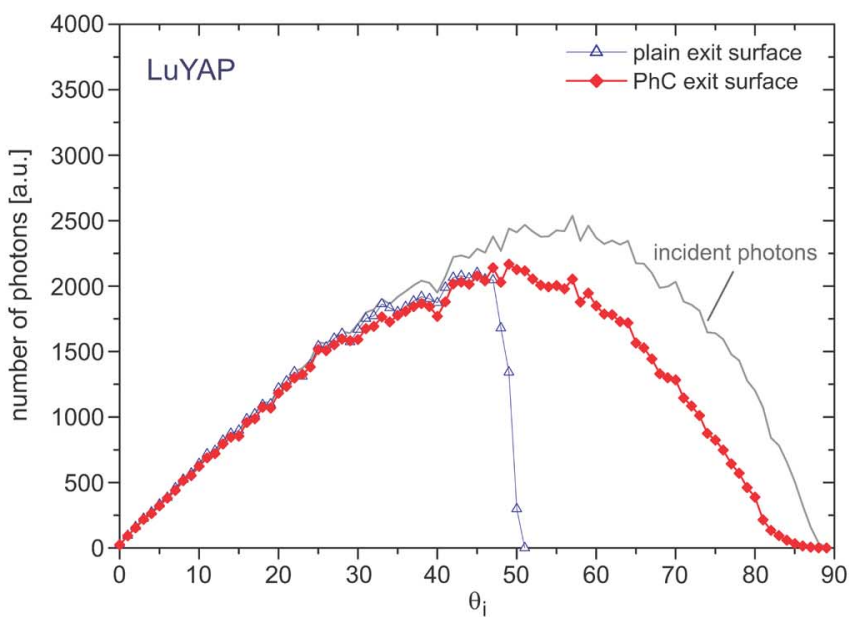

(a)

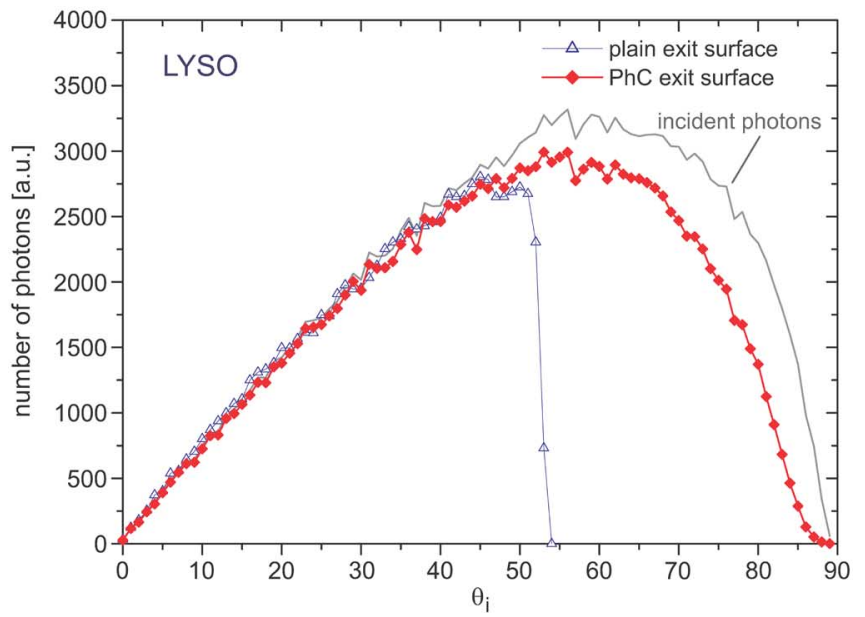

(b)

Fig. 7. Angular distribution of all extracted photons for (a) LuYAP and (b) LYSO and $a / \lambda=0.8$. Scintillator dimensions and $\mathrm{PhC}$ slab parameters are as in Fig. 6(a) and (b).

This behavior can be explained by the angular distribution of the incoming photons: Assuming that the wrapping and its associated crystal face are separated by a layer of air, a photon will hit the wrapping only if its angle relative to this face is smaller than the angle of total reflection for the crystal-air interface, $\theta_{\text {tot,air }}$. For the face opposite to the coupling face, losses are generally small as photons with $\theta_{i}<\theta_{\text {tot,air }}$ will hit the wrapping only once between each incidence on the coupling face. However, even for photons with $\theta_{i}>90^{\circ}-\theta_{\text {tot,air }}$ which experience many reflections at the side faces and therefore, can hit the wrapping several times before reaching again the coupling face, losses are moderate as only photons that fulfil

$$
\tan ^{2}\left(90^{\circ}-\theta_{i}\right)+\tan ^{2} \phi_{f} \leq \tan ^{2} \theta_{\text {tot, air }}
$$

will be able to hit the wrapping, where $\phi_{f}$ is the polar angle of the photon relative to the face. Taking LuYAP $\left(\theta_{\text {tot,air }} \approx 31^{\circ}\right)$ as example, the considerations above imply that the value of $\rho$ is only relevant for photons with $\theta_{i} \geq 65^{\circ}$; even at $\theta_{i}=$ $70^{\circ}$, where the number of incident photons is already rapidly declining, only $50 \%$ of the photons are affected by a reduction in $\rho$.

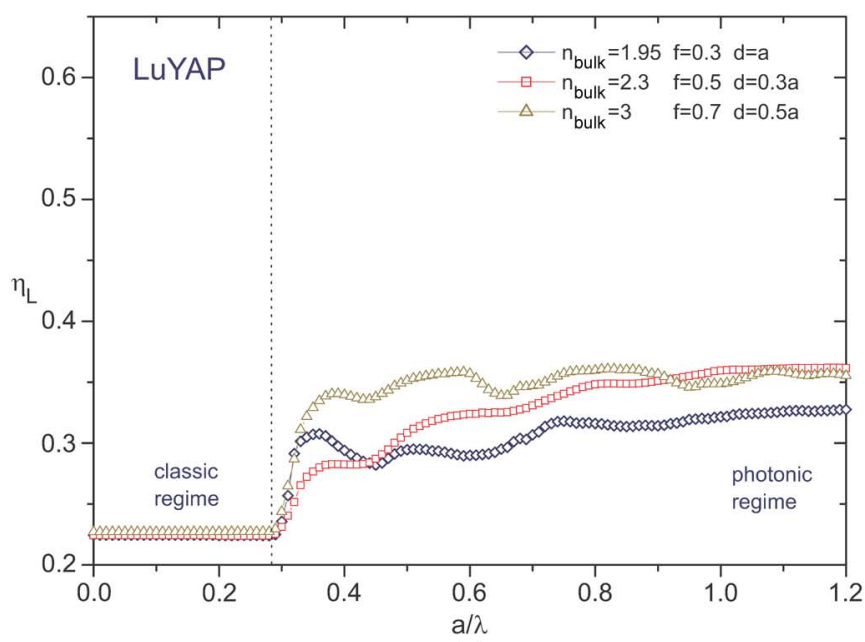

(a)

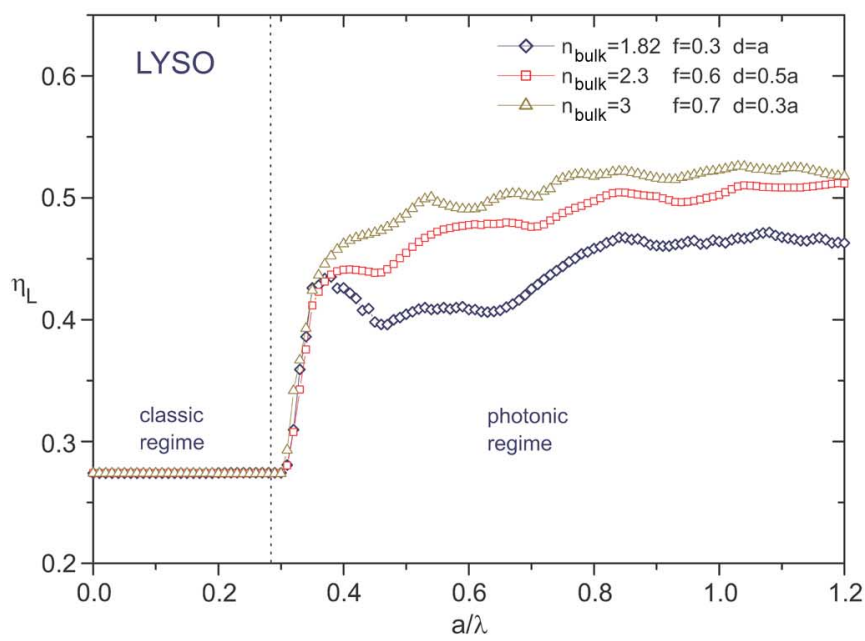

(b)

Fig. 8. Dependence of $\eta_{L}$ on $a / \lambda$ for several PhC-scintillator combinations. (a) LuYAP; (b) LYSO. Curves are shown for combinations of $f$ and $d$ for which $\eta_{L}$ was found to reach a maximum value at the given $n_{\text {bulk }}$. Scintillator dimensions are $2 \times 2 \times 8 \mathrm{~mm}^{3}$ in both cases.

Clearly, the gain in $\eta_{L}$ should only be seen as approximate due to the assumptions of the model, which contradict to some extent the realistic assumptions of the LITRANI model. This can also be seen by the fact that the values of $\eta_{L}$ obtained for the classic regime differ by $\approx 10 \%$ to $15 \%$ from the values determined by LITRANI simulations of crystals with plain exit surface [13]. This difference is mostly due to the non-consideration of scattering and of a wavelength-dependent $\alpha_{\mathrm{abs}}$ in the simple model which both can either lead to an improvement or a reduction of $\eta_{L}$. Still, the results of the simulations serve well in showing the positive influence of a $\mathrm{PhC}$ coupling face on the light extraction. After all, this positive influence is also illustrated by the large increase of the luminosity of LEDs that was achieved in recent years and is, by a good part, due to the use of $\mathrm{PhCs}$ and $\mathrm{PhC}$ slabs to enhance light extraction [14]-[19].

\section{A. Practical Realization of a Scintillator With PhC Coupling Face}

The calculated large gains in $\eta_{L}$ observed with $\mathrm{PhC}$ coupling faces raise the question if such a system can be realized 


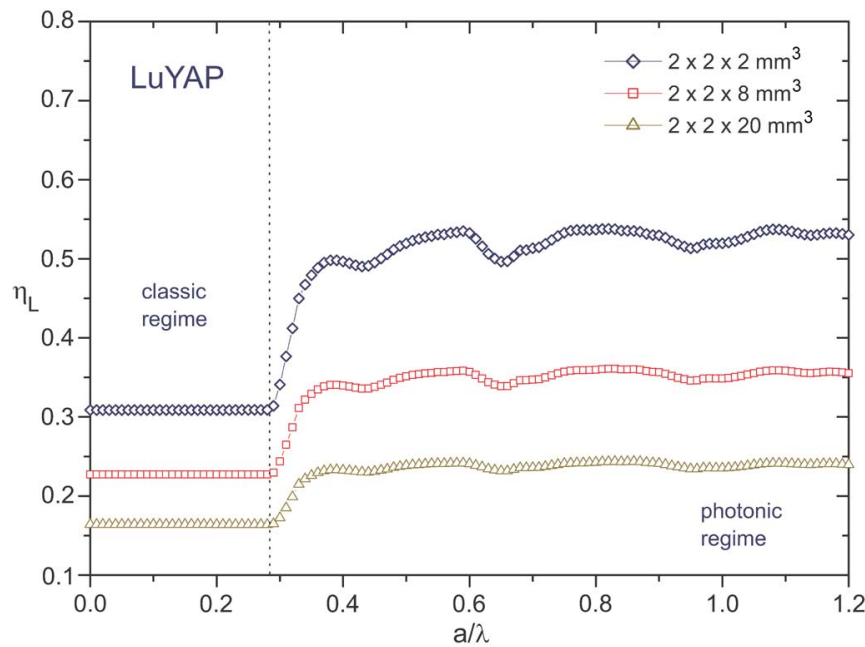

Fig. 9. Dependence of $\eta_{L}$ on $a / \lambda$ for LuYAP scintillators with PhC coupling face and for various scintillator dimensions. The calculation of $\eta_{L}$ is based on a PhC slab with $n_{\text {bulk }}=3, f=0.7$ and $d=0.5 a$.

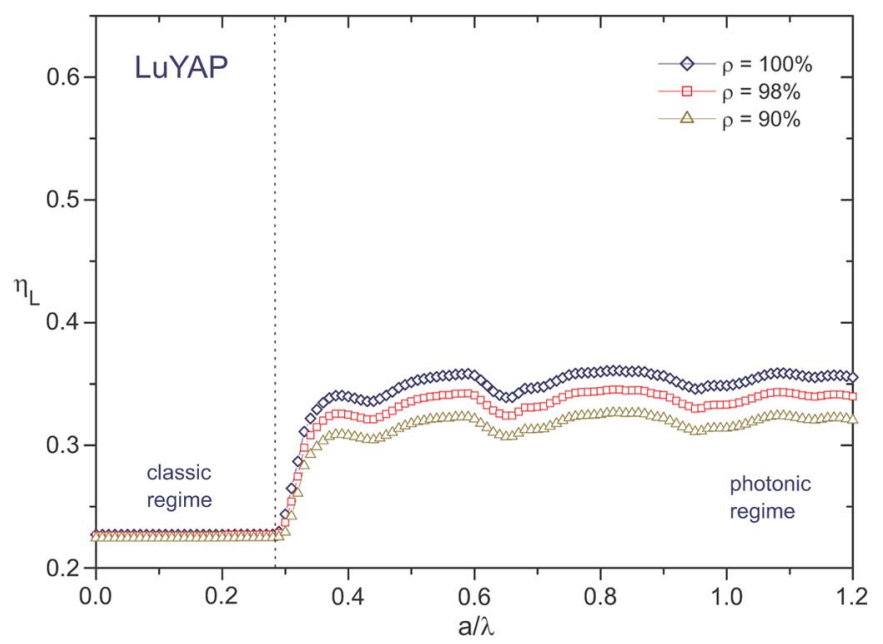

Fig. 10. Dependence of $\eta_{L}$ on $a / \lambda$ for $2 \times 2 \times 8 \mathrm{~mm}^{3}$ LuYAP scintillators with $\mathrm{PhC}$ coupling face and with various wrappings of different reflectivities $\rho$. $\mathrm{PhC}$ slab parameters are as in Fig. 9.

experimentally. In the past 20 years, a number of techniques have been employed for $\mathrm{PhC}$ fabrication, involving classical UV and X-ray lithography [20], holographic methods [21], and mask-less methods such as self-assembly [22], selective area vapor epitaxy [23], or direct imprinting methods using a mold [24]. The most common approaches to produce $\mathrm{PhCs}$ are lithographic methods that use focused electron or ion beams. In both cases, the $\mathrm{PhC}$ bulk layer and a photoresistive mask are deposited on top of the $\mathrm{PhC}$ layer. The photoresistive is then exposed by the electron or ion beam and later moved to a developer where the exposed or non-exposed parts of the resist are removed. After developing the resist, the lithographic $\mathrm{PhC}$ structure is transferred to the $\mathrm{PhC}$ layer by reactive ion etching. Since these processes are routinely used industrial techniques, these production methods have also the potential to allow for a cost-effective production of samples for detector use.
Basically, a scintillator with $\mathrm{PhC}$ coupling face can be realized in two ways. The simplest possibility is to imprint the $\mathrm{PhC}$ structure directly onto the scintillator coupling face without additional $\mathrm{PhC}$ layer, which would correspond to the simulated case that the PhC bulk material has the same refractive index as the scintillator itself. However, direct imprinting is problematic due to technological restrictions and does not yield the gain in $\eta_{L}$ observed for a high- $n$ bulk material [Fig. 8(a) and (b)]. More promising therefore seem to be approaches where the $\mathrm{PhC}$ is contained in a high refractive index layer that is deposited onto the coupling face of the crystal. Such layers are easy to fabricate with modern equipment and the imprinting processes are well understood.

The selection of possible bulk materials is restricted to materials with $\alpha_{\mathrm{abs}} \leq 10^{3}-10^{4} \mathrm{~cm}^{-1}$ in the wavelength range of emission as otherwise, too many photons are lost due to absorption in the $\mathrm{PhC}$ slab. A promising material for $\mathrm{PhC}$ fabrication appears to be $\mathrm{TiO}_{2}$ which exhibits high transmission at wavelengths $>370 \mathrm{~nm}$ and a refractive index that can be varied from $\approx 1.8$ to 2.5 by suitable choice of the deposition parameters [25], [26]. Candidate materials with bulk refractive indexes around and above 3 are $\mathrm{Si}$ and $\mathrm{GaP}$, which however exhibit a strongly increasing $\alpha_{\text {abs }}$ for $\lambda<400 \mathrm{~nm}$ [7], [27]. Both materials are therefore probably suited only for scintillators that emit at larger wavelengths, as e.g., LuAG. Other possible candidate materials include $\mathrm{SiN}$ and $\mathrm{Al}_{x} \mathrm{Ga}_{1-x} \mathrm{~N}$ [28], [29]. For a given material choice, the optimum values of $f, d$ and $a / \lambda$ can then be identified by maximizing $\eta_{L}$; the exact lattice parameters then follow from the emission wavelength $\lambda$ of the scintillator.

\section{CONCLUSION}

The impact of photonic crystal (PhC) slabs on the light extraction from the heavy inorganic scintillators LuYAP and LYSO has been reviewed. Simulations with a scattering-matrix algorithm show that a periodic pattern of air holes with lattice constant $a \approx \lambda$ changes the light extraction properties of the scintillator exit surface significantly, allowing for the extraction photons that hit the exit surface under an angle that is larger than the angle of total reflection of a plain surface. Calculating the number of extracted photons with a simplified model of the photon propagation in the scintillator shows that a scintillator with PhC coupling face has a higher $\eta_{L}$ compared to a scintillator with plain exit surface. By comparison of $\eta_{L}$ determined for $\mathrm{PhC}$ slabs with different properties, it is shown that the gain in $\eta_{L}$ depends on the ratio $a / \lambda$ and the structural parameters of the $\mathrm{PhC}$ slab. The gain in $\eta_{L}$ is higher for $\mathrm{PhC}$ slabs with high- $n$ bulk material and decreases with the scintillator dimensions perpendicular to the $\mathrm{PhC}$ coupling face; for $2 \times 2 \times 8 \mathrm{~mm}^{3}$ LuYAP and LYSO crystals, the gain can reach values up to $60 \%$ and $100 \%$, respectively. Due to the approximations and idealizations of the model, these gains in light collection efficiency should be seen only as approximate values of what can be expected in practical applications and are likely to be achieved only with specular reflectors with high $\rho(>90 \%)$ and $\mathrm{PhC}$ bulk materials with $\alpha_{\text {abs }} \leq 10^{3}-10^{4} \mathrm{~cm}^{-1}$ in the wavelength range of emission of the scintillator. 


\section{ACKNOWLEDGMENT}

The authors would like to thank F.-X. Gentit from CEA Saclay and X. Letartre, C. Seassal and J.-L. Leclercq from INL, Lyon.

\section{REFERENCES}

[1] E. Yablonovitch, "Inhibited spontaneous emission in solid-state physics and electronics," Phys. Rev. Lett., vol. 58, pp. 2059-2062, 1987.

[2] J. Sajeev, "Strong localization of photons in certain disordered dielectric superlattices," Phys. Rev. Lett., vol. 58, pp. 2486-2489, 1987.

[3] M. Kronberger, E. Auffray, and P. Lecoq, "Probing the concepts of photonic crystals on scintillating materials," IEEE Trans. Nucl. Sci., vol. 55, no. 3, pp. 1102-1106, 2008.

[4] E. Auffray, P. Bruyndonckx, O. Devroede, A. Fedorov, U. Heinrichs, M. Korjik, M. Krieguer, C. Kuntner, C. Lartizien, P. Lecoq, S. Leonard, C. Morel, J. B. Mosset, C. Pedrini, A. Petrosyan, U. Pietrzyk, M. Rey, S. Saladino, D. Sappey-Marinier, L. Simon, M. Streun, S. Tavernier, J. M. Vieira, and K. Ziemons, "The ClearPET project," Nucl. Instrum. Methods A, vol. 527, pp. 171-174, 2004.

[5] P. Lecoq and J. Varela, "Clear-PEM, a dedicated PET camera for mammography," Nucl. Instrum. Methods A, vol. 486, pp. 1-6, 2002.

[6] S. G. Tikhodeev, A. L. Yablonskii, E. A. Muljarov, N. A. Gippius, and T. Ishihara, "Quasiguided modes and optical properties of photonic crystal slabs," Phys. Rev. B, vol. 66, 2002, ID: 045102.

[7] J. Humlicek, M. Garriga, M. I. Alonso, and M. Cardona, "Optical spectra of $\mathrm{Si}_{x} \mathrm{Ge}_{1-x}$ alloys,” J. Appl. Phys., vol. 65, pp. 2827-2832, 1989.

[8] A. J. Wojtowicz, W. Drozdowski, D. Wisniewski, J. L. Lefaucheur, Z. Galazka, Z. Gou, T. Lukasiewicz, and J. Kisielewski, "Scintillation properties of selected oxide monocrystals activated with Ce and Pr," Opt. Mater., vol. 28, pp. 85-93, 2006.

[9] F. X. Gentit, "Litrani: A general purpose Monte Carlo program simulating light propagation in isotropic and anisotropic media," [Online]. Available: http://gentit.home.cern.ch/gentit/litrani

[10] NIST XCOM, Photon Cross Sections Database [Online]. Available: http://physics.nist.gov/PhysRefData/Xcom/Text/XCOM.html

[11] M. Kronberger, "Improving the light extraction from heavy inorganic scintillators" Ph.D. dissertation, TU Vienna, Vienna, Austria, 2008 [Online]. Available: http://cdsweb.cern.ch/record/1105938

[12] Y. A. Berdnikov, V. V. Grebenshchikov, V. F. Kosmach, I. E. Leonov, V. M. Samsonov, O. Y. Khrushcheva, and A. P. Shishlo, "Special features of light collection processes in heavy-crystal-based scintillation detectors," Instrum. Exp. Tech., vol. 44, pp. 466-471, 2001.

[13] M. Kronberger, E. Auffray, and P. Lecoq, "Determination of the absolute light yields of LuYAP and LYSO," presented at the IEEE Nucl. Sci. Symp. Med. Imag. Conf. 2008, Dresden, Germany, 2008, Poster N02-353.

[14] A. A. Erchak, D. J. Ripin, S. Fan, P. Rakich, S. D. Joannopoulos, E. P. Ippen, G. S. Petrich, and L. A. Kolodziejski, "Enhanced coupling to vertical radiation using a two-dimensional photonic crystal in a semiconductor light-emitting diode," Appl. Phys. Lett., vol. 78, pp. 563-565, 2001.
[15] H. Y. Ryu, Y. H. Lee, R. L. Sellin, and D. Bimberg, "Over 30-fold enhancement of light extraction from free-standing photonic crystal slabs with InGaAs quantum dots at low temperature," Appl. Phys. Lett., vol. 79, pp. 3573-3575, 2001.

[16] M. Rattier, H. Benisty, R. P. Stanley, J.-F. Carlin, R. Houdre, U. Oesterle, C. J. M. Smith, C. Weisbuch, and T. F. Krauss, "Towards ultrahigh-efficiency aluminium oxide microcavity light-emitting diodes: Guided mode extraction by photonic crystals," IEEE J. Sel. Topics Quantum Electron., vol. 8, no. 2, pp. 238-247, 2002.

[17] Y. J. Lee, S. H. Kim, J. Huh, G. H. Kim, Y. H. Lee, S. H. Cho, Y. C. Kim, and Y. R. Do, "A high-extraction-efficiency nanopatterned organic light-emitting diode,” Appl. Phys. Lett., vol. 82, pp. 3779-3781, 2003.

[18] M. Zelsmann, E. Picard, T. Charvolin, E. Hadji, M. Heitzmann, B. Dal'Zotto, M. E. Nier, C. Seassal, P. Rojo-Romeo, and X. Letartre, "Seventy-fold enhancement of light extraction from a defectless photonic crystal made on silicon-on-insulator,' Appl. Phys. Lett., vol. 83, pp. 2542-2544, 2003.

[19] A. David, H. Benisty, and C. Weisbuch, "Optimization of light-diffracting photonic-crystals for high extraction efficiency LEDs," J. Display Technol., vol. 3, no. 2, pp. 133-148, 2007.

[20] C. Cuisin, Y. Chen, D. Decanini, A. Chelnokov, F. Carcenac, A. Madouri, J.-M. Lourtioz, and H. Launois, "Fabrication of three-dimensional microstructures by high resolution X-ray lithography," $J$. Vac. Sci. Technol. B, Microelectron. Process. Phenom., vol. 17, pp. 3444-3448, 1999.

[21] M. Campbell, D. N. Sharp, M. T. Harrison, R. G. Denning, and A. J. Turberfield, "Fabrication of photonic crystals for the visible spectrum by holographic lithography," Nature, vol. 404, pp. 53-56, 2000.

[22] A.-P. Hynninen, J. H. J. Thijssen, E. C. M. Vermolen, M. Dijkstra, and A. Van Blaaderen, "Self-assembly route for photonic crystals with a bandgap in the visible region," Nature Mater., vol. 6, pp. 202-205, 2007.

[23] L. Yang, J. Motohisa, J. Takeda, and T. Fukui, "Promising low-damage fabrication method for the photonic crystals with hexagonal or triangular air holes: Selective area metal organic vapor phase epitaxy," Opt. Express, vol. 13, pp. 10823-10832, 2005.

[24] S. Y. Chou, C. Keimel, and J. Gu, "Ultrafast and direct imprint of nanostructures in silicon," Nature, vol. 417, pp. 835-837, 2002.

[25] F. Gracia, F. Yubero, J. P. Holgado, J. P. Espinos, A. R. GonzalezElie, and T. Girardeau, " $\mathrm{SiO}_{2} / \mathrm{TiO}_{2}$ thin films with variable refractive index prepared by ion beam induced and plasma enhanced chemical vapor deposition," Thin Solid Films, vol. 500, pp. 19-26, 2006.

[26] H. J. Frenck, W. Kulisch, M. Kuhr, and R. Kassing, "Deposition of $\mathrm{TiO}_{2}$ thin films by plasma-enhanced decomposition of tetraisopropyltitanate," Thin Solid Films, vol. 201, pp. 327-335, 1991.

[27] D. E. Aspnes and A. A. Studna, "Dielectric functions and optical parameters of Si, Ge, GaP, GaAs, GaSb, InP, InAs, and InSb from 1.5 to 6.0 eV," Phys. Rev. B, vol. 27, p. 1009, 1983.

[28] J. F. Muth, J. D. Brown, M. A. L. Johnson, Z. Yu, R. M. Kolbas, J. W. Cook, and J. F. Schetzina, "Absorption coefficient and refractive index of GaN, AlN and AlGaN alloys," MRS Internet J. Nitride Semicond. Res., vol. 4S1, 1999, ID G5.2.

[29] R. Wolf, K. Wandel, and B. Gruska, "Low-temperature ICPECVD of silicon nitride in $\mathrm{SiH}_{4}-\mathrm{NH}_{3}-\mathrm{Ar}$ discharges analyzed by spectroscopic ellipsometry and etch behaviour in KOH and BHF," Surf. Coat. Tech., vol. 142, pp. 786-791, 2001. 